\title{
Kernos
}

Revue internationale et pluridisciplinaire de religion grecque antique

$28 \mid 2015$

Varia

\section{La dea Ecate e i luoghi di passaggio}

Una protettrice dalla quale proteggersi

\section{Nicola Serafini}

\section{Q OpenEdition}

1 Journals

\section{Edizione digitale}

URL: http://journals.openedition.org/kernos/2331

DOI: $10.4000 /$ kernos.2331

ISSN: 2034-7871

\section{Editore}

Centre international d'étude de la religion grecque antique

\section{Edizione cartacea}

Data di pubblicazione: 1 ottobre 2015

Paginazione: 111-131

ISBN: 978-2-87562-055-2

ISSN: 0776-3824

\section{Notizia bibliografica digitale}

Nicola Serafini, «La dea Ecate e i luoghi di passaggio », Kernos [En ligne], 28 | 2015, mis en ligne le 01 octobre 2017, consulté le 19 avril 2019. URL : http://journals.openedition.org/kernos/2331; DOI : 10.4000/kernos.2331

Questo documento è stato generato automaticamente il 19 aprile 2019

Kernos 


\title{
La dea Ecate e i luoghi di passaggio
}

\author{
Una protettrice dalla quale proteggersi
}

\author{
Nicola Serafini
}

Ringrazio il lettore anonimo di Kernos per i preziosi suggerimenti, accanto alla prof. Paola ANGELI BERNARDINI, le cui attente letture hanno stimolato numerose e utili discussioni. Una prima versione di queste pagine fu vista, anni fa, anche dal prof. Claude CALAME, al quale esprimo qui la mia gratitudine, mentre più di recente anche il prof. Robert PARKER ha letto e commentato questo lavoro stimolando la mia riflessione su numerosi punti problematici.

\section{Ecate nelle triodoi e la liminalità nella concezione antica}

1 Negli ultimi anni la dea Ecate ha attirato l'attenzione di numerosi interpreti, e un buon numero di contributi è stato di recente dedicato al suo legame con i luoghi liminali e di passaggio, come ad esempio gli incroci, le porte, e gli ingressi in generale ${ }^{1}$. A dispetto di tale impegno, tuttavia, una maggiore attenzione diacronica sarebbe stata auspicabile: il profilo della dea Ecate nell'antica Grecia subisce nei secoli profonde e radicali mutazioni, perdendo caratteri arcaici e acquisendone sempre di nuovi, in un continuo processo che dura da Esiodo sino alla tarda antichità. Non solo tale dimensione diacronica non può in alcun modo essere sottostimata, ma addirittura essa sembra essere la più corretta chiave di lettura per comprendere la dea Ecate e le pratiche cultuali a lei indirizzate: nello studio di tale figura divina occorre, infatti, seguire i varî momenti di questo lungo e complesso processo, perché è proprio in tale direzione che a questo punto è ormai indispensabile rivolgersi ${ }^{2}$.

2 La presenza della dea Ecate nei luoghi liminali, inoltre, assume un'ampia gamma di manifestazioni cultuali, ciascuna delle quali racchiude una sfumatura diversa. I molteplici valori di questi costumi rituali conferiscono a Ecate sfere d'influenza fra loro assai diverse, dalla dimensione della purificazione alla tutela delle transizioni, passando per la funzione apotropaica dei suoi simulacri. L'immagine che ne emerge è quella di una divinità ambigua ${ }^{3}$, di una protettrice dalla quale occorre anche proteggersi. 


\section{Incroci e luoghi di passaggio}

Le fonti greche descrivono una serie di rituali compiuti negli incroci: dai deîpna offerti alla dea Ecate, allo scarico dei rifiuti domestici ritenuti potenzialmente contaminanti ( oxythymia), dalla performance di riti magici ${ }^{4}$, fino alla deposizione, alla lapidazione o al rogo dei cadaveri virtualmente pericolosi in quanto macchiati di colpe specifiche ${ }^{5}$. Il ruolo particolare della triodos è del resto presente anche nel mito, specificamente nella vicenda di Edipo, in cui l'uccisione di Laio ha luogo proprio in un trivio ${ }^{6}$.

4 Non sarà necessario, in questa sede, trattare nel dettaglio questioni generali e metodologiche relative ai luoghi di passaggio. Mi limito dunque solamente a ricordare le opinioni in merito di S.I. Johnston, secondo la quale i punti liminali sono percepiti in maniera del tutto particolare, persino potenzialmente pericolosa, per l'impossibilità di essere ricondotti a categorie ben precise, vale a dire per il fatto di non appartenere né all'uno né all'altro dei due dominî da essi separati. Ad esempio, la soglia non è né dentro né fuori dall'edificio, così come l'incrocio tecnicamente non è parte di nessuna delle strade che vi s'intersecano ${ }^{7}$. Ogni punto liminale (che sia la soglia della casa, un crocicchio, o le frontiere vere e proprie), è connotato dalla dimensione dell' 'alterità' rispetto a ciò che lo circonda. A rigor di termini, tuttavia, potrebbe non sembrare corretto considerare allo stesso modo sia i luoghi di passaggio sia i punti liminali ${ }^{8}$. In effetti, non va dimenticato che la soglia di una casa è un punto di passaggio sic et simpliciter, mentre non lo è invece il trivio: quest'ultimo implica una dimensione di scelta, estranea al semplice luogo di passaggio. Fatta questa premessa generale, tengo a precisare che nel presente contesto non ho ritenuto opportuno distinguere le due tipologie di liminalità (frontiere da un lato e luoghi di passaggio dall'altro) proprio perché entrambe riconducibili alla caratteristica comune dell'alterità: ciò che qui interessa è proprio la dimensione di estraneità che contraddistingue questi luoghi, che siano di passaggio o di frontiera, sempre e comunque - in un certo qual modo - 'distaccati' ontologicamente dal contesto, quando non fisicamente. Permane sempre e comunque un'alterità sostanziale che separa il luogo di passaggio da ciò che lo circonda, ed è tale alterità che qui interessa.

\section{Ecate Enodia}

5 La dea Ecate è intimamente connessa ai crocicchi, da cui l'epiclesi di $\tau \rho ı \delta \tilde{i} \tau 1 \zeta^{9}$. In maniera più generale, ma forse più indicativa e pertinente, le è spesso conferito anche l'epiteto di

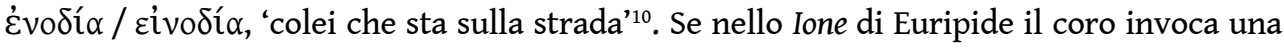

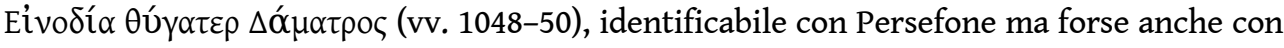
Ecate (cfr. Call. fr. 466 Pf.), ancora prima in un frammento del Catalogo delle donne esiodeo si nomina una Artemide Einodia (fr. 23a, 26 M.-W.), identificabile con ogni probabilità con la dea Ecate (cfr. fr. 23b M.-W.).

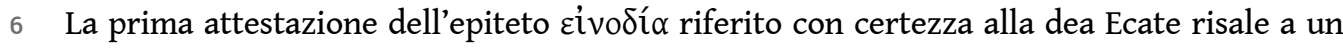
frammento dei Rhizotomoi di Sofocle che riporta un'invocazione a Elio (fr. 535 R.), nella quale la dea appare per la prima volta connotata da aspetti spaventosi - come ad esempio la capigliatura di serpenti -, manifestazioni di una dimensione oscura e temibile che da

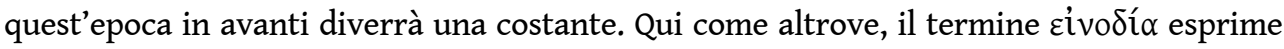
la connessione di Ecate con le strade, specificamente con i crocicchi, nominati al quarto verso. Inoltre, un passo dell'Elena di Euripide, opera datata al 412, dimostra che già alla 
fine del $\mathrm{V}$ sec. il teonimo Enodia poteva essere utilizzato come una variazione perfettamente equipollente del nome della dea Ecate, perlomeno in ambito ateniese ${ }^{11}$.

7 Enodia, però, oltre a essere un'epiclesi di Ecate ${ }^{12}$, era anche il teonimo di una dea locale venerata in Tessaglia, il cui culto si sarebbe poi diffuso anche ad Atene, Sicione e Argo, stando alle indicazioni di Pausania (II, 10, 7; 23, 5). Per quanto riguarda la tessala En (n)odia, divinità misteriosa e assai poco nota, raffigurata spesso a cavallo contrariamente a Ecate ${ }^{13}$-, disponiamo di scarsissimi particolari ${ }^{14}$ : a livello iconografico $\mathrm{i}$ suoi principali attributi sono il cavallo, il cane e la fiaccola ${ }^{15}$. Il suo santuario più antico era situato al di fuori del centro di Fere, presso la porta Nord e non lontano dalla necropoli $\mathrm{N}$, sulla strada verso Larissa, e delle iscrizioni ritrovate in loco documentano che la dea era qui venerata assieme a Zeus Thaulios-Aphrios: l'antichità del culto è provata dal ritrovamento di piccoli manufatti databili fino all'età geometrica, e i dati archeologici permettono di seguire gli sviluppi di tale sito. Così, ad esempio, sappiamo che sul finire dell'età arcaica fu eretto un grande tempio dorico, andato però distrutto sul finire del IV sec. a.C., sùbito dopo rimpiazzato, attorno al 300 a.C. da un nuovo tempio, ancora più maestoso, edificato sulle rovine di quello precedente. Un altro luogo di culto dedicato alla dea Enodia si trovava nei pressi della necropoli o di Fere, dove la dea era associata a Demetra e Zeus Meilichios: l'associazione cultuale con Zeus Meilichios, testimoniata del resto anche a Larissa grazie a un'epigrafe (IG IX 2, 578), richiama da vicino quella di Selinunte, dove Ecate e Zeus Meilichios erano entrambi venerati nello stesso santuario, quello di Demetra Malophoros, specificamente adibito al culto di divinità ctonie ${ }^{16}$. Infine, il ritrovamento di una stele votiva e di un altare dedicato a Enodia in punti differenti da quelli già menzionati, documentano che verosimilmente dovevano esistere altri luoghi a lei sacri nella città (o nei pressi) di Fere. Tuttavia, fatta esclusione per i pochi particolari già menzionati e per la sua natura di Wegegöttin, testimoniata dal nome stesso ${ }^{17}$, conosciamo poco altro su questa oscura divinità, che sembra presentare forti legami con il mondo infero, ma che rimane comunque piuttosto misteriosa ai nostri occhi.

Sebbene A. Zografou sia tornata recentemente a difendere l'autonomia locale di Enodia rispetto a Ecate ${ }^{18}$, l'influenza esercitata da questa divinità locale sullo sviluppo della Ecate panellenica non può essere misconosciuta. Le argomentazioni avanzate a riguardo da P. Marquardt, infatti, mostrano in maniera convincente come l'acquisizione di caratteri ctonî da parte di Ecate sia coeva proprio alle prime attestazioni ad Atene del culto della tessala Enodia ${ }^{19}$. È proprio nella città attica, infatti, che Ecate è rappresentata per la prima volta come trimorfa da Alcamene, ed è sempre ad Atene, attorno agli stessi anni, che nei testi drammatici ella va assumendo tutti quei connotati che fino allora erano a lei totalmente estranei. Tutto ciò accade proprio nell'epoca in cui inizia a essere attestato ad Atene il culto di una divinità locale tessala, che, inoltre, presentava già in origine alcuni tratti in comune con Ecate, come ad esempio la qualità di Wegegöttin, rendendo così ancora più agevole l'assimilazione della dea locale minore con quella già più famosa, anche se non ancora panellenica, cantata fin dai tempi di Esiodo.

Difficile stabilire, sulla base dei documenti in nostro possesso, se nel caso di Ecate ed Enodia si possa parlare di 'sincretismo', di 'assimilazione' o di 'identificazione' ${ }^{20}$. In ogni caso sembra ormai chiaro che qualcosa deve essere accaduto con l'arrivo ad Atene di Enodia. Nella fattispecie, si potrebbe pensare a una identificazione iniziale fra le due divinità, cui avrebbe fatto séguito una graduale assimilazione, testimoniata dall'assunzione del teonimo di Enodia come epiclesi di Ecate, fino a che quest'ultima non finì con l'assorbire quasi completamente la dea locale, al punto di assumerne il nome. Non 
sembra tuttavia possibile stabilire un momento ben preciso per un fenomeno simile: non solo, ma non sembra neppure legittimo volerlo fare. Quello che ha investito la dea Ecate $\mathrm{fu}$ un lento e graduale processo di acquisizione di nuove prerogative, in cui questa divinità è andata ottenendo attributi sempre più connessi alla sfera dell'oscurità e della magia $^{21}$, assieme a un profilo - per così dire - 'ctonio' ${ }^{22}$, che da quest'epoca in avanti non si separerà più dalla sua figura.

\section{Statuette di Ecate nei crocicchi: gli hekataia}

10 La presenza protettiva della dea Ecate negli incroci e la sua funzione di guida erano assicurate in molti modi. Innanzitutto, in questi luoghi esistevano dei piccoli tempietti a lei dedicati, ma soprattutto erano diffusissime le statuette raffiguranti la dea tricorpore,

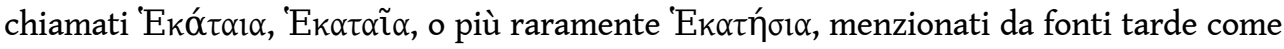

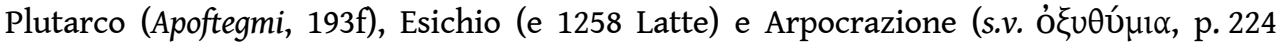
Dindorf). Un notevole numero di tali manufatti è giunto sino a noi, grazie a ritrovamenti archeologici sparsi in quasi tutto il mondo greco: tali statuette spaziano dalla fine del $\mathrm{v}$ sec. alla tarda età imperiale, e solitamente raffigurano la dea triplice, sottoforma di tre giovani fanciulle addossate sulla schiena contro una colonna centrale, la cui resa artistica mostra di norma uno stile arcaizzante ${ }^{23}$.

11 Almeno dalla fine del v sec., sulla scia della Epipyrgidia di Alcamene ${ }^{24}$, le statue della dea collocate nei trivî erano comunemente trimorfe, come se ognuna delle facce della dea dovesse sorvegliare una delle tre vie che si congiungevano nell'incrocio (Cfr. Ovidio, Fasti I, 141-142): infatti, sembra che la figura tricorpore di Ecate fosse in genere intesa come intimamente legata al suo culto nelle triodoi ${ }^{25}$, ipotesi seguita anche da alcuni studiosi moderni ${ }^{26}$. Già gli antichi, tuttavia, tendevano a interpretare il trimorfismo della dea anche in funzione dei tre regni su cui si pensava avesse potere (terra / Ade, cielo e mare), e sui quali già nella Teogonia Ecate ha una parte di sovranità ${ }^{27}$. In ogni caso, nelle fonti letterarie di epoca ellenistica e imperiale continuano a permanere numerosi echi del

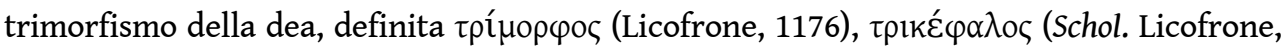

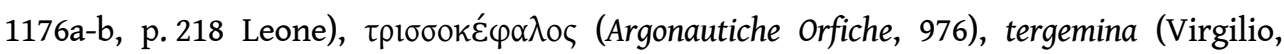
Eneide IV, 511), triceps (Ovidio, Metamorfosi VII, 194), triplex (Ov., Fast. I, 387), triformis (Ov., Met. VII, 94, 177; Seneca, Medea, 7). Tale peculiarità non solo attraversa tutta l'antichità, ma addirittura non abbandonerà più la dea, e la accompagnerà attraverso l'età moderna fino ai giorni nostri e al Neo-Paganesimo contemporaneo ${ }^{28}$.

\section{Ecate nei trivî: i rituali descritti nelle fonti letterarie}

12 Le fonti antiche hanno tramandato la menzione di alcuni rituali in onore della dea Ecate celebrati nei crocicchi. In questi punti liminali non erano semplicemente presenti statue o tempietti a lei dedicati, ma la dea vi era altresì onorata, come afferma eloquentemente

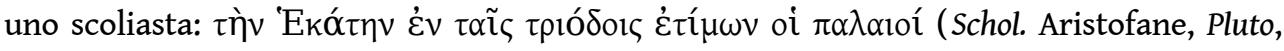
594a $\alpha$, p. 105 Chantry). Ciò è confermato da un altro scolio allo stesso verso che riporta la medesima notizia, anche in questo caso utilizzando una formula ( intuire la desuetudine all'epoca dello scoliasta della pratica descritta (Sch. Ar., Pl., 594a $\beta$, p. 105 Chantry). 
13 Riguardo alla venerazione della dea Ecate nelle triodoi, uno scolio a Teocrito è l'unica fonte antica a fornirci un vero e proprio mito eziologico, connettendo la nascita stessa della dea con i crocicchi. Lo scoliasta riporta, infatti, una versione secondo cui la dea, figlia di Zeus e Ferèa, fu deposta dalla madre in un crocicchio, per poi essere trovata e allevata da uno dei pastori di Ferete (Schol. Teocr., II, 35 / 36a, p. 278, 11-4 Wendel). Tale versione, oltre alla singolare genealogia, offre un'inedita coordinata geografica, perché se il Ferete di cui parla lo scoliasta fosse lo stesso fondatore di Fere in Tessaglia ${ }^{29}$, questo mito localizzerebbe la nascita della dea proprio in questa regione, andando a integrare il dossier sui rapporti fra Ecate e la Tessaglia, dunque con Enodia. Il racconto riportato dal commentatore, di conseguenza, oltre a connettere la nascita di Ecate ai crocicchi, le fornisce anche una collocazione geografica mai attestata altrove.

\section{I 'pasti di Ecate'}

14 La presenza della dea nei crocicchi sembra essere un punto fisso nella credenza antica, almeno a partire dal v secolo. Numerose fonti menzionano la pratica dell'offerta di cibo in onore di Ecate, i cosiddetti $\delta \varepsilon \tilde{\imath} \pi \nu \alpha{ }^{~ E k \alpha ́ ́ \tau \eta \varsigma, ~ i ~ q u a l i ~ e r a n o ~ i m b a n d i t i ~ e ~ o f f e r t i ~ a ~ q u e s t a ~}$ divinità nei crocicchi. La prima attestazione dell'offerta di cibo alla dea risale a un frammento di Sofocle: non sembra possibile, tuttavia, stabilire a quale dramma appartenesse. In ogni caso nel breve frammento i cibi stessi, plausibilmente offerti alla

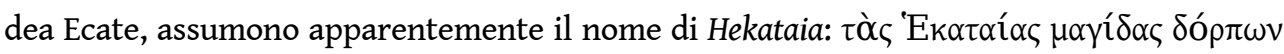
(fr. 734 R.). Il sostantivo $\mu \alpha \gamma i ́ \delta \varepsilon \varsigma$, presente nel breve frammento, può indicare focacce e cibi in senso lato, ma da una notizia di Polluce sappiamo che questo era il termine solitamente utilizzato nel caso di 'pasti sacri' (Polluce, X, 81, II p. 213 Bethe: i $\varepsilon \rho \grave{\alpha ~} \delta \varepsilon \tilde{\pi} \pi v \alpha$ ), mentre Fozio ancora più specificamente lo riconduce ai 'pasti di Ecate' (Fozio, s.v. $\mu \alpha \gamma$ í $\delta \varepsilon \varsigma$, II p. 530 Theodoritis). Il frammento sofocleo in questione purtroppo non permette ulteriori analisi per la sua stringatezza, ma attesta in maniera significativa che all'epoca era già in uso la pratica dell'offerta di cibi alla dea Ecate.

15 Anche Sofrone attesta l'usanza, elencando in un frammento i varî tipi di pani e focacce offerti alla dea (fr. 26 K.-A.). Non è molto più esaustiva nemmeno la menzione che di tale offerta rituale fa Aristofane nel Pluto, quando nell'agone fra Cremilo e la Povertà a un certo punto sono ricordati proprio i $\delta \varepsilon \tilde{\imath} \pi \nu \alpha$ consacrati alla dea Ecate (Ar., Pl., 594-597). Il

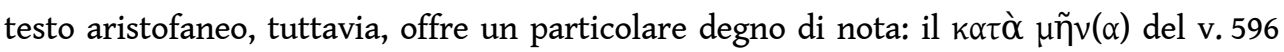
specifica la mensilità di tali offerte, particolare su cui torneremo.

Per ora, vale la pena notare la contrapposizione fra ricchi e poveri: il commediografo dice chiaramente che l'impossessarsi del cibo offerto alla dea era segno di estrema povertà. Gli scholia vetera alla commedia precisano lo svolgimento dell'insolita pratica per cui i ricchi offrivano i 'pasti' alla dea mentre i poveri, dopo aver ingerito quelle offerte, affermavano fosse stata la stessa Ecate a mangiarle (Schol. Ar., Pl., 594c $\alpha-\beta$, p. 105 Chantry). Non diversamente, Demostene utilizza in un'orazione proprio l'argomento dei 'pasti' di Ecate

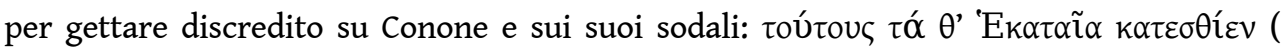
Contro Conone, 39). Oltre a connotare un grado di miseria non indifferente, il cibarsi di queste offerte denotava anche una biasimevole noncuranza verso le pratiche religiose, se

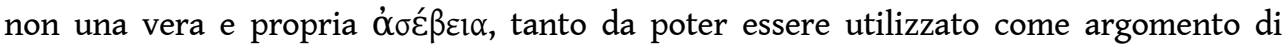
accusa. Qualche secolo dopo, nell'Inno a Demetra di Callimaco, quando Erisittone è colpito da una fame tremenda per volere della dea Demetra, con la sua insaziabilità finisce col prosciugare le dispense del padre Triopa, e si vede costretto a elemosinare cibo nei 
crocicchi, probabilmente in attesa delle offerte per Ecate (v. 113-115) ${ }^{30}$. Anche qui sembra essere suggerito che la miseria costringesse alcuni a nutrirsi dei cibi consacrati alla dea. Infine, anche Luciano menziona questa pratica cultuale allorché, all'inizio dei Dialoghi dei morti, Diogene incarica Polluce di risalire sulla terra e di andare a prendere il cinico Menippo per condurlo nell'Ade. Il filosofo, tuttavia, dovrà prima premunirsi di molti lupini e di un 'pasto di Ecate', qualora ne trovasse uno incustodito in un crocicchio (Luc., Dial. Morti I, 1; cfr. Luc., Cataplus VII, 1-4). Non è ben chiara la menzione dei $\delta \varepsilon \tilde{\pi} \pi v \alpha$ in tale contesto: è possibile che si voglia alludere appunto alla miseria del filosofo e al fatto che debba anch'egli ricorrere al furto di tali cibarie consacrate alla dea ${ }^{31}$.

Mentre solitamente le fonti nominano la dea Ecate come unica destinataria di tali offerte

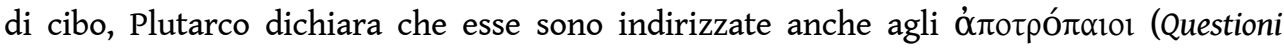
Conviviali, 708f), cioè a divinità - non meglio specificate - che proteggevano dal male e lo tenevano lontano. Questo inedito accostamento potrebbe permettere di inserire i 'pasti di Ecate' fra i rituali di tipo apotropaico, come una richiesta affinché la dea protegga gli uomini dal male e dai pericoli insiti nei crocicchi: questa è la conclusione alla quale giunge S.I. Johnston ${ }^{32}$, in direzione opposta rispetto agli studiosi precedenti, che invece consideravano Ecate come la principale minaccia di tali luoghi. L'assunto dominante, infatti, è sempre stato quello secondo cui la dea Ecate sarebbe stata essa stessa uno dei pericoli relativi ai trivî, se non il più spaventoso. I rituali che avevano luogo in quegli spazî dovevano essere intesi proprio a scacciare gli spiriti maligni e la dea Ecate in primis, piuttosto che a ingraziarsela per guadagnarne la protezione.

In realtà, nessuno dei due punti di vista appare risolutivo: a questo punto sembra opportuno abbandonare prospettive troppo intransigenti, soprattutto anche alla luce delle tendenze più recenti, che hanno ben mostrato la duttilità e la mutevolezza delle divinità greche in base ai luoghi e alle occasioni. Non ha più senso, ormai, tentare di isolare un solo e unico aspetto del potere di Ecate per stabilire quale sia la 'vera identità' della dea: Ecate non è o una minaccia, o una protettrice, Ecate è entrambe le cose insieme. È sì una divinità apotropaica, in un certo senso, poiché protegge i mortali dal male e dagli attacchi degli spiriti; tuttavia, è essa stessa un pericolo per i mortali, che cercano di tutelarsi dalla pericolosità della dea con offerte - direi quasi - profilattiche. Gli stessi 'pasti di Ecate' partecipano dell'ambiguità della dea: da un lato offerta profilattica per salvaguardarsi da Ecate, dall'altro richiesta di protezione affinché la stessa Ecate protegga i mortali.

Nel caso di questa dea in particolare, ma è un discorso valido in generale anche per le altre figure divine greche, è tramontata l'epoca delle interpretazioni univoche, che tentavano di ricondurre a un solo aspetto, o a una sola prerogativa, tutte le manifestazioni cultuali dedicate a una divinità. Per quanto riguarda Ecate, come già anticipato, non solo non si può procedere in maniera binaria, per antitesi: occorre bensì comprendere che la dea racchiude entrambi gli opposti, e così i rituali a lei offerti sono spesso interpretabili in maniere fra loro antitetiche, senza che per forza di cose debba prevalere un aspetto piuttosto che un altro.

20 Tornando ai dati cultuali, Aristofane, come abbiamo visto, riguardo alla frequenza dei

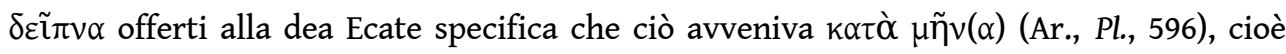

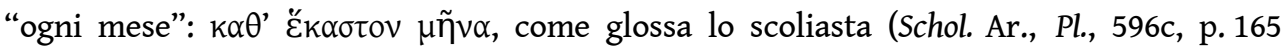
Chantry). Altre fonti concordano sulla regolarità dell'offerta mensile dei 'pasti di Ecate', $\mathrm{e}$ sono ancora più precise riguardo alla cadenza di tali donazioni: Ateneo, ad esempio,

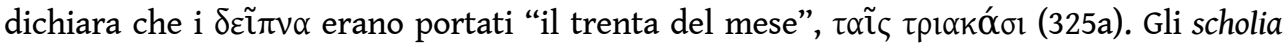


vetera ad Aristofane collimano con tale affermazione, asserendo che le offerte alla dea si

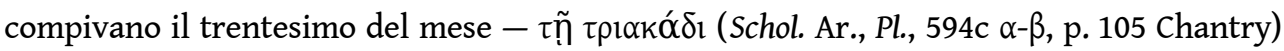
-: poiché il passaggio da un mese all'altro coincide con il novilunio, questi riti si eseguivano evidentemente in quella notte, come del resto non manca di notare lo

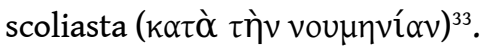

Nella notte fra il vecchio mese e il nuovo, dunque in un momento di transizione cronologica, la dea Ecate era venerata negli incroci, punto liminale e di passaggio per eccellenza. Dimensione spaziale e dimensione temporale convergono nel suggerire la connessione della dea con la sfera del passaggio: tutelante sia la transizione fisica da una strada a un'altra attraverso il trivio, sia quella temporale, consistente nel passaggio da un mese al successivo, dalla luna vecchia a quella nuova. Lo storico Filocoro (FGrHist. $328 \mathrm{~F}$ 86), inoltre, afferma che ad Atene le offerte erano inviate ai crocicchi anche il 16 del mese, "perché in quel giorno la luna, mentre tramonta, è sorpresa dal sorgere del sole e così il cielo è illuminato doppiamente» ${ }^{34}$ : il 16 del mese corrisponde alla notte del plenilunio. Sebbene non sia specificato se tale offerta fosse appannaggio della dea Ecate, lo possiamo

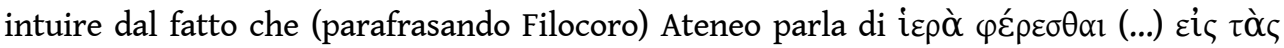

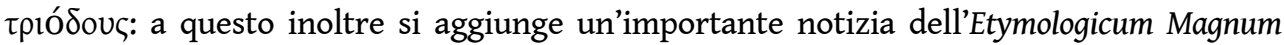

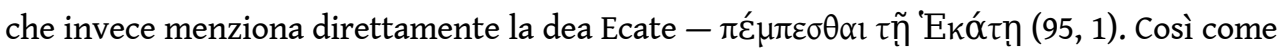
i rituali che avevano luogo nella notte del novilunio erano connotati dalla dimensione di 'liminalità temporale' dovuta al passaggio dal vecchio mese a quello nuovo, allo stesso modo anche la notte del plenilunio va intesa come denotante la transizione fra due fasi temporali ${ }^{35}$.

Tralasciando, per ragioni di spazio, una descrizione dettagliata degli alimenti che componevano i 'pasti di Ecate', mi limiterò a rilevare qui la grande varietà di cibi offerti alla dea: da pani e focacce di vario genere, sino a uova e pesce, in particolare triglie. Per concludere, il rito appare iscritto nella dimensione del culto privato, in assenza di sacerdoti o officianti scelti a celebrare l'offerta, che, stando a quanto affermano le fonti, sembra consistere nella semplice deposizione di cibo nei trivî, senza alcuna cerimonia ufficiale da seguire. L'unica prescrizione rituale da rispettare riguardava la data dell'offerta, che doveva avvenire nel trentesimo giorno del mese, in concomitanza con la notte di novilunio, in una dimensione di transizione temporale fra un mese e l'altro.

\section{I rifiuti scaricati nei crocicchi: katharsia e oxythymia}

Le offerte incruente caratterizzate dalla consacrazione dei 'pasti di Ecate' non sono le uniche forme rituali eseguite nei trivî. In questi luoghi, infatti, erano scaricati anche i resti delle cerimonie di purificazione che avevano luogo privatamente nella dimensione domestica $^{36}$ : essendo una sorta di 'non-luogo', i crocicchi appaiono come i punti più appropriati per depositare i rifiuti indesiderati espulsi dalla società, giacché questi siti erano parzialmente dissociati dalla città e dalle strade stesse, godendo di uno status a sé3 ${ }^{37}$.

Secondo le informazioni offerte da numerose fonti, con il termine oxythymia si qualificavano i resti - potenzialmente 'contaminati' - che rimanevano dopo i riti di purificazione compiuti in ambito domestico. Il dato rilevante sul quale concordano le fonti è che queste rimanenze, come anticipato, erano depositate nei trivîi ${ }^{38}$. Anche Plutarco menziona i materiali di scarto dei riti purificatorî che erano lasciati nei crocicchi, chiamandoli però katharsia (Questioni Romane, 280c, 290d), dove il termine potrebbe includere gli stessi oxythymia. I commentatori, infatti, definiscono gli oxythymia 
con l'utilizzo di termini quali katharsia o katharmata con cui evidentemente ci si riferisce sempre ai residui dei riti di purificazione (cfr. Eustazio, ad Od. p. 1935, 10-11).

Il significato e la composizione degli oxythymia rimangono piuttosto difficili da definire, poiché le fonti non sono concordi. Arpocrazione sembra intendere in maniera equipollente katharsia e oxythymia (s.v. ỏ $\xi 0 \theta u ́ \mu l \alpha$, p. 224, 5 Dindorf), senza però specificarne la composizione, mentre l'Etymologicum Magnum definisce gli oxythymia come i rifiuti dei riti sacri $(626,45-46)$. I katharsia erano quindi i resti che rimanevano dopo le pratiche di purificazione compiute privatamente nelle case. Questi rimasugli potevano anche essere fumigati nella casa stessa, come attestano alcune fonti tarde (Plutarco, Quest. Conv., 709a. Cfr. anche Schol. Esch., Coef., 98a). Tutto ciò che era stato preparato per tali riti, ma non era stato effettivamente adoperato, prendeva il nome di katharmata. Fra questi rientravano anche le rimanenze che non potevano essere bruciate, per cui erano

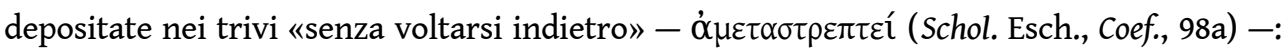
Ateneo includeva in tale categoria anche l'acqua e il sangue che restavano dopo i

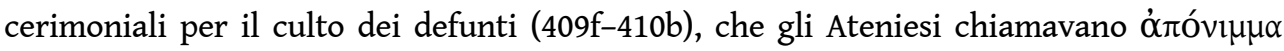
(409f).

Il dato decisivo è offerto da Arpocrazione (s.v. ỏ $\xi \theta \theta u ́ \mu \nu \alpha$ ), il quale precisa che Didimo

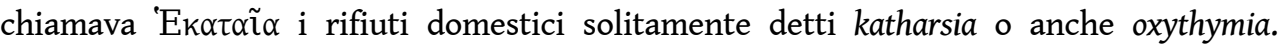
L'informazione è del resto corroborata da una notizia dell' Etymologicum Magnum (626, 4446), dove si dice significativamente che tali materiali potevano anche essere chiamati

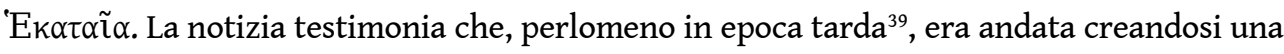

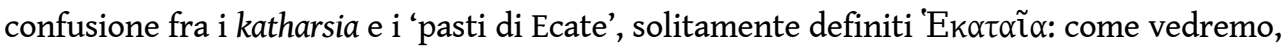
anche in Plutarco si assiste a tale 'fusione' fra i due differenti rituali, allorché egli connette i sacrifici di cani sia con i deîpna di Ecate sia con i riti di purificazione (Quest. Rom., 280c, 290d).

\section{Sacrifici di cani in onore della dea Ecate}

Per quanto riguarda il sacrificio cruento di cani in onore di Ecate, la prima testimonianza in ordine temporale è Sofrone (fr. 8 Kaibel), che in uno dei suoi mimi menzionava tali

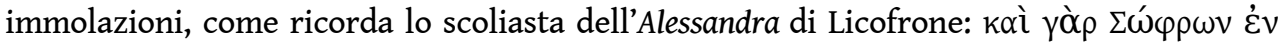

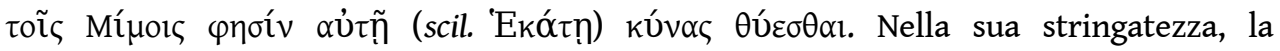
dichiarazione documenta l'antichità di una pratica che purtroppo è passata sotto silenzio dalla maggior parte delle fonti coeve a noi giunte. L'unico altro testo anteriore al tardo ellenismo in cui si faccia riferimento a tali sacrifici è costituito da un frammento di Aristofane (fr. 209 K.-A.), che è anche la fonte più importante risalente all'epoca classica. Il commediografo, infatti, è il primo a menzionare le triodoi come luogo di tali offerte e specifica che la vittima è un cagnolino di colore bianco, piuttosto che nero, come sarebbe stato lecito aspettarsi ${ }^{40}$. Sul colore della vittima, infatti, dopo aver affermato che gli efebi lacedemoni sacrificavano nel Phoibaion un cucciolo di cane al dio Enialio, Pausania aggiunge di non essere informato di altri sacrifici di cani praticati dai Greci, a eccezione dei Colofonî, che sacrificano alla dea Ecate una cagnolina nera (III, 14, 9).

Un sacrificio di cuccioli neri, inoltre, è descritto anche nelle Argonautiche Orfiche (934-987)

${ }^{41}$, databili a un'epoca molto tarda, forse addirittura al IV sec. d.C. Nel testo 'orfico', Mopso istruisce gli Argonauti che, dovendo introdursi nel recinto dove il dragone sorveglia il vello d'oro, invocano Ecate per avere la sua protezione: Orfeo, che è l'officiante, scava un bothros $^{42}$ triangolare e lo riempie con varî tipi di legname; dopodiché vi pone sopra delle 
figurine impastate con farina. A quel punto, compie il sacrificio vero e proprio,

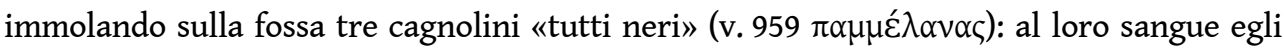
mescola diversi tipi di erbe, scelte personalmente da Medea, e dopo averlo riversato nello stomaco delle vittime sacrificali, le pone sulla sommità della pira, mentre il resto delle interiora è collocato intorno al bothros. Orfeo suona quindi «il bronzo sonoro» e prega: all'invocazione fa séguito la teofania delle Erinni, Tisifone, Aletto e Megera, cui segue quella di Pandora e infine quella mostruosa della stessa dea Ecate. Le Erinni appaiono portando delle torce, e la pira nel bothros si accende improvvisamente da sola e inizia ad ardere.

Dopo Aristofane per alcuni secoli le fonti in nostro possesso tacciono su questo rito in onore di Ecate, che torna a essere menzionato solamente negli scolî. In questi ultimi, tuttavia, il sacrificio di cani è ormai assimilato all'offerta dei 'pasti di Ecate', sì che nelle fonti tarde le due pratiche vengono a fondersi in una sola consuetudine rituale. Nell'immaginario comune si pensava che la dea si cibasse appunto di cani, pertanto questi ultimi erano a lei sacrificati intendendoli come deîpna, venendo a sostituire le offerte di cibo attestate dalle fonti classiche. In uno scolio a Teocrito, infatti, sono menzionate le offerte di cani, e questi ultimi sono intesi dal compilatore come elementi stessi dei 'pasti di Ecate' (Schol. Teocr., II, 11 / 12d, p. 273, 1-2 Wendel).

Data l'esiguità delle fonti a riguardo non è possibile stabilire se anche in epoca classica il sacrificio di cani in onore di Ecate fosse inteso già come pasto rituale della dea, ma bisogna certo tener conto che a quell'epoca le offerte dei deîpna erano solitamente composte di cibo vero e proprio, come abbiamo avuto modo di vedere in precedenza. Ecco dunque l'importanza di uno studio diacronico. L'analisi differenziale delle fonti letterarie permette di cogliere aspetti che altrimenti passerebbero sotto silenzio.

Se negli scolî è già percepibile un qualche mutamento nella concezione di tale rituale, è solo con Plutarco - e non prima - che tale processo può dirsi compiuto. Il rituale del periskylakismos, che a detta di Plutarco era eseguito da «tutti i Greci», consisteva nell'uccisione di un cucciolo di cane. Lo svolgimento di tale pratica è descritto nelle Questioni Romane $e^{43}$, dove il sacrificio di cuccioli di cane è legato alla dimensione della purificazione, e la dea Ecate è chiaramente connessa a tali riti. Questo legame è in realtà piuttosto raro, se non inedito prima di Plutarco. In un altro passo lo storico di Cheronea testimonia che alla sua epoca i tre rituali costituiti dai 'pasti di Ecate', dai riti di purificazione domestica, e dai sacrifici di cani, avevano finito col fondersi in un'unica manifestazione rituale. Se in precedenza $\mathrm{i}$ tre aspetti erano ben distinti, nella testimonianza di Plutarco, invece, il cane è offerto come deîpnon alla dea in occasione dei riti di purificazione: «Gli antichi non credevano che il cane potesse mantenersi puro. Infatti, non è offerto in sacrificio ad alcuno degli olimpî, quantunque sia inviato come pasto per la ctonia Ecate nei trivî, dove costituisce una parte delle offerte apotropaiche e purificatorie» (Quest. Rom., 290d) ${ }^{44}$.

Offerta di deîpna alla dea nei crocicchi, sacrificio di cani, dimensione purificatoria: questi tre elementi appaiono ormai inseparabili. Il testo di Plutarco, infatti, è perspicuo nel riferire il sacrificio del cane alla sfera dei riti apotropaici e purificatorî ${ }^{45}$, cui la dea Ecate non era mai accostata nelle fonti anteriori all'età imperiale. La stessa divinità sembra ora chiaramente connessa alla dimensione purificatoria, ragion per cui i crocicchi da lei presieduti sono divenuti la sede di offerte simili. Il sacrificio cruento è a questo punto ritenuto una componente del 'pasto' di Ecate: tale mutamento appare evidente dal testo di Plutarco, e non prima. 

ruolo decisivo. Non si può certo ignorare lo sviluppo che ha investito nel corso dei secoli il sacrificio che qui interessa. Se queste offerte in epoca classica presero il nome di deîpna perché costituite proprio da cibo, è possibile che questo nome nella consuetudine linguistica abbia continuato a denotare ogni offerta alla dea Ecate, anche quando questi sacrifici non erano più costituiti da vivande vere e proprie. Anzi, è possibile che il sacrificio di cani in onore della dea, già presente nel rituale del v sec., sia poi andato ad affiancare - o persino a sostituire - quello di pane e formaggi, e sia poi stato incluso nel novero dei deîpna, poiché questo termine denotava ormai in maniera molto ampia tutto ciò che era offerto alla dea nei trivî.

\section{La dea davanti alle porte: Ecate Propylaia}

La dea Ecate era presente anche all'entrata delle case, in un punto che per definizione è un luogo di passaggio. In virtù della sua tutela delle porte e delle entrate, Ecate era quindi chiamata Propylaia ${ }^{46}$ : questa dea presiedeva gli ingressi in senso lato, e in molti casi era collocata all'entrata di santuarî dedicati ad altre divinità, come ad esempio a Selinunte o a Eleusi ${ }^{47}$. Inoltre, la presenza della dea all'entrata delle case richiama una dimensione apotropaica che la accosta a figure come quelle della Gorgone o di Dioniso, le cui maschere erano sovente appese nelle case o nelle entrate, in virtù della loro presunta capacità di allontanare gli influssi malefici.

Due testimonianze letterarie del v sec. alludono specificamente alla presenza di Ecate davanti alle porte. Il primo è un frammento eschileo contenente un canto corale (fr. 388 R.), del quale non è tuttavia possibile stabilire il dramma di appartenenza. Il frammento attesta la presenza di Ecate davanti all'entrata delle case dei re, e nonostante la brevità del testo tramandato si può comunque percepirne il tono solenne ${ }^{48}$. A livello linguistico,

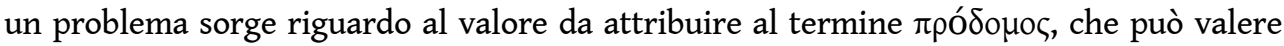
sia come sostantivo sia come aggettivo ${ }^{49}$ : nel frammento eschileo, tuttavia, sembra più plausibile interpretare il termine come aggettivo riferito direttamente alla dea, invocata come 'colei che sta davanti alle dimore regie'.

Il secondo testo di epoca classica menzionante la presenza 'fisica' di Ecate davanti alle porte e agli ingressi, è un passo delle Vespe di Aristofane in cui Filocleone esamina la possibilità di eseguire i processi davanti alle case e di costruire negli ingressi dei piccoli tribunali "ovunque, davanti alle porte, simili a hekataia" (v. 804). Questo passo aristofaneo è spesso citato come documento principale per la presenza degli hekataia davanti agli ingressi delle case, sebbene nella fattispecie sia possibile che subentri un certo grado di esagerazione comica, allorché il testo sembra affermare che davanti a ogni casa immancabilmente ci fosse un'edicola dedicata a Ecate. Il termine 'Ekó́toıov, come spiegano gli scolî, comprendeva sia i tempietti dedicati alla dea, sia le semplici statue che la raffiguravano ${ }^{50}$. Sulla posizione di queste edicole all'ingresso delle case è esplicito lo scoliasta a Teocrito (Schol. Teocr., II, 35 / 36a, p. 278, 15 Wendel), così come lo sono sia Plutarco (Apoft., 193f) sia Esichio ( $\varepsilon 1258$ Latte).

37 Restano infine da menzionare altri due passi di Aristofane in cui il poeta allude agli hekataia. Il primo, dall'interpretazione problematica nonché controversa, si trova all'inizio della Lisistrata, quando la protagonista e Calonice attendono con impazienza l'arrivo delle altre donne ateniesi nel luogo stabilito per l'incontro, e si domandano 


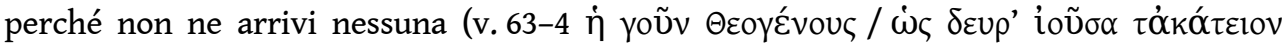
n̆ ai contributi degli specialisti, sono sostanzialmente due ${ }^{51}$. Nel primo caso, la battuta alluderebbe alla proverbiale dedizione femminile per il vino: la moglie di Teagene ha "preso il volo", ha "alzato la vela", ma la frase può suonare anche come se dicesse che ha

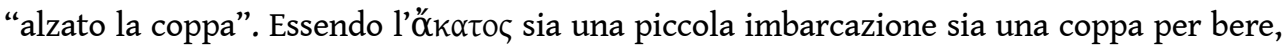
ci sarebbe qui un gioco di parole attestato peraltro anche in un frammento di Epicrate (fr. 9, 1 K.-A.), che significherebbe dunque qualcosa come "ha alzato il gomito". In alternativa,

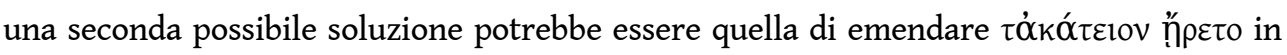

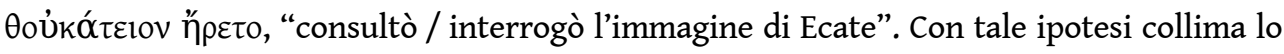

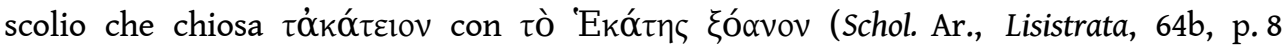
Hangard), ma anche la Suda ( $\theta 80$ Adler; cfr. anche $\varepsilon 361$ Adler).

Un'altra allusione agli hekataia, infine, è offerta da Aristofane in un passo delle Rane, in cui il coro biasima una serie di soggetti e intima loro di «allontanarsi dal coro degli iniziati» (v. 370), fra i quali si allude a qualcuno che «imbratta i simulacri di Ecate cantando nei cori ciclici» (v. 366). Gli scolî affermano che Aristofane allude qui al ditirambografo Cinesia $^{52}$. Non è ben chiaro, ciò nonostante, se il commediografo si riferisca nella fattispecie a una cronica indisposizione intestinale da cui era affetto il poeta Cinesia ${ }^{53}$, o piuttosto a un poema lirico dedicato alla dea Ecate, in cui il ditirambografo aveva forse offerto un ritratto poco lusinghiero della divinità (cfr. Schol. Vet. Ar., Rane, 366d).

\section{Conclusioni}

La presenza della dea Ecate nei luoghi liminali, come abbiamo visto, assumeva una notevole varietà di manifestazioni ${ }^{54}$, ciascuna delle quali racchiudeva una sfumatura diversa a livello religioso. Alla fine di questo (rapido) esame dei rituali relativi ai crocicchi e alle entrate, infatti, la prima qualità che occorre rimarcare è proprio la varietà delle manifestazioni e dei luoghi in cui possiamo rintracciare la dea Ecate in rapporto ai luoghi di passaggio. Ben visibile a chiunque, la dea godeva anche di numerosissime piccole edicole o tempietti edificati dagli stessi privati davanti alle abitazioni, la cui soglia era da lei sorvegliata e protetta ${ }^{55}$. Persino nei santuarî intitolati ad altre divinità, Ecate poteva vantare la presenza di piccoli templi in suo onore o di statue che la raffiguravano, collocati in buona parte dei casi nelle entrate. Per quanto riguarda lo sviluppo diacronico della dea, non possiamo non ammettere che il mutamento nella figura divina di Ecate quale emerge dalle fonti letterarie sia stato un processo contemporaneamente attivo su più fronti, senza che nessuno dei varî aspetti sia peraltro confluito in una vera e propria rottura con la tradizione precedente. Un'accattivante ipotesi sarebbe quella dell'assimilazione della tessala Enodia a Ecate: allo stato attuale delle nostre conoscenze archeologiche ed epigrafiche, tuttavia, non è possibile dimostrare l'assimilazione di queste due divinità, perlomeno non ancora, anche se mi sembra evidente che debba essersi verificato un qualche 'incontro' fra Ecate ed Enodia.

In definitiva, alla luce delle pratiche e delle credenze analizzate, si potrebbe affermare che Ecate è una protettrice benevola, ma che al contempo costituisce essa stessa una minaccia per i mortali. Non si tratta di dare o togliere, premiare o punire, come fanno comunemente le altre divinità: Ecate è una figura che protegge dagli spiriti, ma è essa stessa un pericolo dal quale occorre proteggersi. Ciò è dimostrato dai rituali compiuti per 
timore della dea e per tenerla lontana: ella stessa è la minaccia, ciò che spaventa i mortali. Eppure, allo stesso tempo, è anche colei che può tenere il pericolo lontano.

41 La differenza rispetto alle altre divinità è sostanziale: esse sono ambivalenti, non ambigue. Per fare un esempio, Ermes può concedere prosperità agli armenti, ma anche decretarne la morìa: tuttavia, ciò non fa di lui una divinità ambigua, bensì ambivalente. È ovvio, e ampiamente risaputo, che le divinità greche hanno la facoltà di dare ma anche di togliere: tuttavia, nel caso di Ecate non è questione di dare o togliere. Si tratta, per così dire, della 'natura' stessa della divinità, la quale è ambigua, e può essere allo stesso tempo sia benevola sia crudele, o entrambe le cose insieme, senza che sia il comportamento umano a suscitarne l'ira. La dea costituisce di per sé un pericolo nei confronti degli uomini, e al contempo è invocata dagli stessi affinché conceda loro la sua protezione. Sin da Esiodo (Teogonia, 411-452), del resto, emergeva questo carattere ambiguo della dea Ecate, connotata da una sorta di 'capricciosità', poiché ella poteva mostrarsi benevola e aiutare i mortali, ma solo chi voleva e quando voleva, oppure anche decidere di non farlo, senza che tale scelta fosse influenzata in alcun modo dal comportamento dei mortali stessi. Le altre figure divine, invece, possono senza dubbio rivelarsi crudeli verso i mortali, ma sempre e solo in séguito a un misfatto o per un atto di empietà (gli esempî sono infiniti: Niobe, Atteone, Agamennone...), non certo per cruccio. In questi casi la divinità agisce solo per ripristinare la giustizia e punire una scelleratezza: dunque, c'è una notevole differenza. Artemide, per fare un nome fra tanti, non costituisce di per sé una minaccia: occorre solo eseguire gli atti di culto prestabiliti, nei modi e nei tempi fissati, per non macchiarsi di empietà, e allo stesso tempo per non oltrepassare i limiti dei mortali pensando di poter competere con una divinità, peccando di hybris. Osservati questi precetti, i soggetti divini non costituiscono più una minaccia per il mortale che, se rispetta le norme religiose, non incorre nell'ira divina.

Il caso di Ecate è diverso. Ecate è una dea ambigua perché rappresenta in sé, a prescindere dalle azioni umane, una minaccia dalla quale guardarsi con rituali profilattici, e allo stesso tempo è anche una protettrice che garantisce ai mortali la tutela dagli attacchi degli spiriti e dai pericoli che popolano i luoghi di passaggio. La sua non è un'ambivalenza, come quella delle altre divinità, che agiscono in differenti maniere pur mantenendo un'unica 'identità': quella di Ecate è ambiguità perché è insita nella natura stessa di tale figura divina, sin dall'epoca di Esiodo. Le divinità greche agiscono in maniera benevola o crudele verso i mortali: Ecate, invece, è a un tempo benevola $e$ crudele verso i mortali.

\section{BIBLIOGRAFIA}

E. CALCATERRA, ' «Per cena alle divinità». Note sul culto privato di Ecate nella Grecia antica', MSS

13 (2005-2006), p. 15-27.

E. CALCATERrA, 'Ecate Signora dei limina. Una rilettura delle fonti più antiche', Mythos 3 (2009),

p. 93-115. 


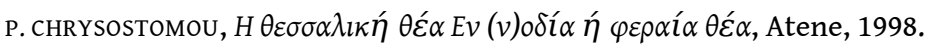

S.G. COLE, Landscapes, Gender and Ritual Space, Berkeley / London / Los Angeles, 2004.

M.W. DICKIE, Magic and Magicians in the Graeco-Roman World, London, 2001.

M. DORATI, 'Teagene e Teogene (Aristoph. Lys. 63 s.)’, QUCC n.s. 72 (2002), p. 77-89.

G. EKRоTH, The Sacrificial Rituals of Greek Hero-Cults in the Archaic to the Early Hellenistic Periods, Liège, 2002.

C.A. FARAONE, 'Household Religion in Ancient Greece', in J. BODEL, S.M. OLYAN (eds.), Household and Family Religion in Antiquity, Malden / Oxford / Carlton, 2008, p. 210-228.

F. FERRARI, L. PRAUSCELLO, 'Demeter Chthonia and the Mountain Mother in a New Gold Tablet from Magoula Mati', ZPE 162 (2007), p. 193-202.

S. FERRI, 'La tripròsopos di Calalzo', RAL s. VIII, vol. V, fasc. 5-6 (1950), p. 330-332.

M.D. FULLERTON, 'The Location and Archaism of the Hekate Epipyrgidia', AA (1986), p. 669-675.

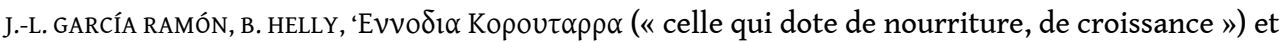
autres divinités kourotrophes en Thessalie', RPh 81 (2007), p. 291-312.

F. GRAF, 'Gods in Greek Inscriptions: Some Methodological Questions', in J.N. BREMMER, A. ERSKINE (eds.), The Gods of Ancient Greece, Edinburgh, 2010, p. 55-80.

S. HALLIWELL, 'Where the Roads Meet: a Neglected Detail in the Oedipus Tyrannus', JHS 106 (1986), p. $187-190$

B. HELLY, 'Consécration d'un enclos funéraire à Ennodia Ilias à Larisa (Thessalie)', Kernos 23 (2010), p. 53-65.

J. HENDERSON (ed.), Aristophanes. Lysistrata, Oxford, 1987.

S.I. JOHNSTON, Hekate Soteira. A Study of Hekate's Roles in the Caldean Oracles and Related Literature, Atlanta, 1990.

S.I. JOHNSTON, ‘Crossroads', ZPE 88 (1991), p. 217-225.

S.I. JOHNSTON, Restless Dead, Berkeley / Los Angeles / London, 1999.

R. KASSEL, C. AUSTIN, Poetae Comici Graeci III. 2, Berlin / New York, 1984.

T. KRAUS, Hekate. Studien zu Wesen und Bild der Göttin in Kleinasien und Griechenland, Heidelberg, 1960.

C. MAINOLDI, 'Cani mitici e rituali tra il regno dei morti e il mondo dei viventi', QUCC 37 (1981), p. 7-41.

P.A. MARQUARDT, 'A Portrait of Hecate', AJPh 102 (1981), p. 243-260.

E. MAzzolA, 'Ecate: solo dea delle donne? La dea nelle testimonianze letterarie dalle origini al III secolo a.C.', Acme 59 (2006), p. 305-318.

M. MONTANARI, N. SERAFINI, Zeus Ammon in Libia, fra culti indigeni e tradizioni greche, Roma (c.d.s.).

C. MORGAN, Early Greek States Beyond the Polis, London / New York, 2003.

A. MOUSTAKA, s.v. «Enodia», LIMC III.1 (1986), p. 743-744.

R. PARKER, Polytheism and Society at Athens, Oxford, 2005. 
R. PARKER, 'Artémis Ilithye et autres : le problème du nom divin utilisé comme épiclèse', in N. BELAYCHE, P. BRULÉ, G. FREYBURGER, Y. LEHMANN, L. PERNOT, F. PROST (eds.), Nommer les dieux, Rennes, 2005b, p. 219-226.

G.B. PELLEGRINI, 'Iscrizioni paleovenete da Làgole di Calalzo (Cadore)', RAL s. VIII, vol. V, fasc. 5-6 (1950), p. 307-329.

G.B. PELLEGRINI, 'Divinità paleovenete', PP 17 (1951), p. 81-94.

V. PIRENNE-DELFORGE, J. SCHEID, 'Qu'est-ce qu'une mutation religieuse?', in L. BRICAULT, C. BONNET (eds.), Panthée: Religious Transformations in the Graeco-Roman Empire, Leiden / Boston, 2013, p. 309313.

N. PUTZERT, ' "Du schnöder Trank aus mitternächt'gem Kraut, dreimal vom Fluche Hekates betaut»: Hekate als Schutzgöttin und Dämonenherrin', in G. UERSCHELN (ed.), «Vielleicht ist die Wahrheit ein Weib... »: Frauengestalten des Mythos im Zwielicht, Köln, 2009.

N. SERAFINI, 'L"'Inno a Ecate" di Esiodo (Theog. 411-452): una falsa definizione', Aevum (ant) n.s. 11 (2011), p. 191-201.

N. SERAFINI, 'I sentieri di Ecate', QUCC n.s. 101 (2012), p. 225-234.

N. SERAFINI, 'Una lekythos ateniese a figure nere: una nuova lettura', Ostraka 21 (2012b), p. 179-187.

N. SERAFINI, "Artemide e i margini, fra eschatiai e kourotrophia: liminalità "topografica" vs. “curotrofica”, Mythos 7 (2013), p. 145-163.

N. SERAFINI, 'La dea Ecate, le torce e le ninfe Lampadi: un frammento di Alcmane da rivalutare (fr. 63 Davies)', QUCC n.s. 104 (133) (2013b), p. 11-22.

N. SERAFINI, 'La dea Ecate in Beozia: un culto-fantasma?', Teiresias 43 (2013c), p. 23-27.

N. SERAFINI, 'La dea Ecate a Cirene fra storia, culto e iconografia (con un catalogo degli hekataia editi e di tre inediti)', in M. LUNI (ed.), Cirene greca e romana, Roma, 2014, p. 107-126.

N. SERAFINI, ‘Antichi dèi, oggi: la Ecate dei Neo-Pagani', Minerva 27 (2014b), p. 1-23.

N. SERAFINI, 'La "rinascita” di una dea greca: la fortuna di Ecate dal Medioevo al Neo-Paganesimo contemporaneo', RCCM 58 (2015), p. 169-192.

N. SERAFINI, 'Il potere di Demetra, fra racconto mitico e cornice rituale: possibili echi del culto cireneo in Callimaco, Inno VI?', in O. MEI, V. PURCARo (eds.), Cirene greca e romana II. Atti del XII Convegno di Archeologia Cirenea (Urbino, 28-29 giugno 2013), Roma, 2015b, p. 14-33.

N. SERAFINI, 'La dea Ecate a Selinunte: una “messaggera” della Malophoros?', in O. MEI, V. PURCARO (eds.), Selinunte nell'antichità: prospettive e ricerche, Atti del Convegno (Urbino 17-18 ottobre 2012), Roma (c.d.s.).

N. SERAFINI, 'Il silenzio come atto rituale, fra culti ctonî e cerimonie magiche', in P. ANGELI BERNARDINI (ed.), Le funzioni del silenzio nella Grecia antica: antropologia, poesia, storiografia, teatro, Atti del Convegno del C.I.S.G.A. (Urbino, 9-10 ottobre 2014), Pisa / Roma (c.d.s.).

N. SERAFINI, 'La povertà nella sfera religiosa greca, tra furto di cibo consacrato ed elemosina rituale', in M. SANTUCCI (ed.), Ploutos \& Polis. Aspetti del rapporto tra economia e politica nel mondo greco, Atti del Convegno (Roma, 20-22 maggio 2013), Roma (c.d.s.).

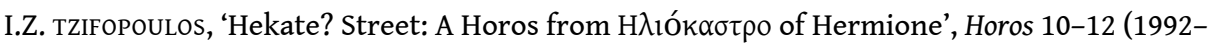
1998), p. 251-258. 
N. WERTH, Hekate. Untersuchungen zur dreigestaltigen Göttin, Hamburg, 2006.

U. V. WILAMOWITZ-MOELLENDORFF, Der Glaube der Hellenen I, Berlin, 1931.

A. zOGRAFOU, 'L'énigme de la triple Hécate de l'entre-deux à la triplicité', in C. BATSCH, U. EGELHAAFGEISER, R. STEPPER (eds.), Zwischen Krise und Alltag, Stuttgart, 1999, p. 57-79.

A. zografou, 'Les repas d'Hécate', in ThesCRA II, Los Angeles, 2003, p. 229-231.

A. ZOGRAFOU, 'Élimination rituelle et sacrifice en Grèce ancienne', in S. GEORGOUDI, R. KOCH PIETTRE, F. SCHмiDT (eds.), La Cuisine et l'Autel, Brepols, 2005, p. 197-213.

A. zografou, Chemins d'Hécate. Portes, routes, carrefours et autres figures de l'entre-deux, Liège, 2010.

\section{NOTE}

1. Tale direzione fu inaugurata da JOHNSTON (1991) - da leggere assieme a JOHNSTON (1999), p. 203$249-$, ma è solo negli anni più recenti che si è assistito all'apparizione di numerosi contributi in merito: ved. ad es. ZOGRAFOU (1999); zOGRAFOU (2003); CALCATERRA (2005-2006); CALCATERRA (2009); e soprattutto ZOGRAFOU (2010). Più in generale, la rinnovata attenzione verso questa divinità è testimoniata dalla pubblicazione di recenti monografie a lei dedicate, per una discussione delle quali si rinvia a SERAFINI (2012).

2. Cfr. in tal senso, seppure in linea generale e non riferito alla dea Ecate, quanto affermato di recente da PIRENNE-DELFORGE, SCHEID (2013), p. 309-313: «L'enjeu des études à venir pourrait être de percevoir à quel moment et selon quelles modalités on assiste, non plus à une évolution qui relève d'une différence de degré dans l'expression, mais à une 'mutation' au sens évoqué plus haut, qui implique une différence de nature dans les actes posés et dans la relation qui s'instaure avec le divin» (p. 313).

3. Di recente, anche PUTZERT (2009) sembra offrire un rapido accenno alla natura ambigua della dea Ecate, tuttavia essa è erroneamente interpretata come una ambivalenza: «Hekate ist als Göttin des Übergangs zwischen hell und dunkel, die Glück und Unglück spendet, die wahre und unwahre Auskunft gibt, ambivalent aufgefaßt worden»(p.66). In realtà, come vedremo, i concetti di 'ambivalenza' e 'ambiguità' non sono da confondere: ved. infra, p. 23. Cfr. già PARKER (2005), p. 20, che definisce Ecate «an ambiguous figure».

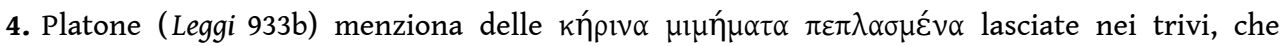
JOHNSTON (1991), p. 223 interpreta come «"voodoo" figures» inerenti a veri e propri riti magici. Pratiche simili sono, in effetti, descritte nei papiri magici (PGM IV, 2943-2966; XXXVI, 256-264), dove si parla di maledizioni eseguite per mezzo di figurine ceree depositate negli incroci, o ancora, dell'iscrizione di defixiones su cocci lasciati in quei luoghi.

5. Lo stesso Platone (Leg. $873 \mathrm{~b}-\mathrm{c}$ ), fornendo 'istruzioni' riguardo al trattamento dei parricidi, afferma che dopo l'esecuzione del colpevole, il suo cadavere debba essere portato in un crocicchio al di fuori della città, dove gli ufficiali dovranno lapidarlo "per purificare la città". Ancora prima, in un frammento del commediografo Eupoli (fr. $132 \mathrm{~K}$.-A. = fr. 120 K.) viene detto

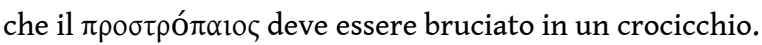

6. HALLIWELl (1986) ha specificamente analizzato il rapporto fra triodos e parricidio nel mito di Edipo.

7. JOHNSTON (1991), p. 217, assieme a JOHNSTON (1990), p. 24-25. Sulla liminalità, in generale, non si possono non ricordare le pagine di COLE (2004), p. 178-180, 185-188, 198-201. Cfr. ora anche S ERAFINI (2013).

8. Come mi ha fatto giustamente notare Claude CALAME. 
9. Cariclide, fr. 1,1 K.-A.; Cornuto, Nat. Deor., 34. Cfr. PGM IV, 1434; IV, 2563; IV, 2724, 2728. Sul legame con i trivî, ved. su tutti zogRAFOU (2010), p. 109-122: a proposito di tale volume, cfr. SERAFINI (2012).

10. Cfr. Johnston (1991), p. 23-24 e MAzzola (2006), p. 311-312. Il legame della dea con le strade è confermato ora dal ritrovamento a Ermione, in Argolide, di un horos in cui è nominata una

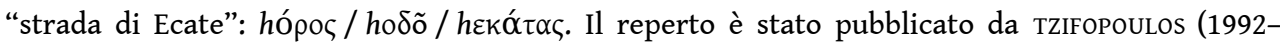
1998).

11. Euripide, Elena, 569-570. In proposito, cfr. KANNNICHT (1969), p. 160: «In Hel.'s Erwiderung ist

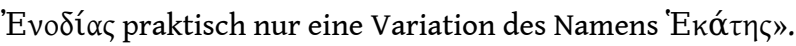

12. Sul fenomeno dei teonimi utilizzati come epiclesi, assai diffuso nel politeismo greco, ved. ora PARKER (2005b). Cfr. anche GRAF (2010), p. 70-71: sul teonimo En(n)nodia, che fuori dalla Tessaglia diventa epiclesi di altre divinità, p 71, n. 73 .

13. Sebbene Ecate sia in qualche caso connessa all'animale, a livello iconografico essa non è mai raffigurata a cavallo, bensì accanto a essi. Cfr. KRAUS (1960), p. 80: «Das Pferd spricht ebenfalls wenig für Hekate». Ancora prima, già perentorio WILAMOWITZ (1931), p. 174: «Hekate reitet nicht».

14. Le uniche fonti letterarie a offrire qualche informazione sulla divinità sono due scolî: Schol. Licofrone, 1180 (p. 219 LEONE) e Schol. Teocrito, II, 36a (p. 278 WENDEL). Cfr. Polieno, VIII, 43 che narra la vicenda di una sacerdotessa tessala di Enodia. Sull'antichità della dea, KRAUS (1960), p. 78 afferma: «Wie alt sie ist, wissen wir freilich nicht». Per qualsiasi approfondimento, si rinvia soprattutto a CHRYsostomou (1998). Tutti i dati disponibili sulla dea Enodia sono stati a suo tempo raccolti e commentati già da KRAUS (1960), p. 77-83. Tuttavia, cfr. anche MOUSTAKA (1986); JOHNSTON (1999), p. 208-209, nonché più di recente MORGAN (2003), p. 135-142; FERRARI, PRAUSCELLO (2007), p. 200-202; GARCíA RAMÓN, HELLY (2007); e HELLY (2010). La forma aggettivale del teonimo, come mi ha giustamente fatto notare Robert PARKER, potrebbe far dubitare sull'indipendenza della dea ed essere forse l'indizio di una storia più complessa: tuttavia, per il momento, il discorso non può essere qui affrontato nel dettaglio.

15. MOUSTAKA (1986), p. 744.

16. Su Selinunte, ved. ora SERAFINI, Selinunte (con ampia bibliografia). Anche altrove, ad esempio in Calcide, Ecate e Zeus sembrano associati a livello cultuale: ved. IG XII 1, 958.

17. Per quanto riguarda la sua prerogativa sui luoghi di passaggio, si noti che piccole statue di Enodia sembra fossero poste all'entrata delle case: ved. ad es. IG IX 2, 575; 577.

18. ZOGRAFOU (2010), p. 123-124.

19. MARQUARDT (1981), p. 252-253 e passim.

20. Sui tali concetti, per una messa a punto teorica del problema del contatto fra divinità differenti, rinvio a MONTANARI, SERAFINI, zeus Ammon, Cap. 1, 'Zeus o Ammone? Aspetti teorici storico-religiosi dell'incontro fra culture differenti: prestiti, identificazioni e sincretismi’ (N. SERAFINI)

21. DICKIE (2001), p. 35 ha ben rilevato il carattere graduale dell'acquisizione, da parte di Ecate, del controllo sugli aspetti magici. Lo studioso, a ragione, nota come tale processo sia graduale e le ragioni oscure: cfr. infra, p. 22.

22. La dicotomia 'ctonio' vs. 'olimpico' non aveva presso i Greci le stesse valenze che noi moderni le attribuiamo. La questione è assai complessa, e non può essere affrontata in questa sede: mi limito dunque a segnalare le considerazioni che ho già espresso in merito altrove, anche con particolare riferimento alla dea Ecate: cfr. ad es. SERAFINI (2012), p. 226, n. 1; e ora SERAFINI, Silenzio, p. 49 , n. 2.

23. Sugli hekataia ved. SERAFINI (2014), con bibliografia anteriore: in particolare, sui caratteri generali e i moduli figurativi di tali statuette, ved. p. 115-18 (cfr. p. 118-26 per un catalogo degli hekataia ritrovati a Cirene, inclusi tre inediti). Per quanto riguarda il trimorfismo della dea Ecate a livello iconografico, e soprattutto il suo sviluppo diacronico, cfr. SERAFINI (2012b), mentre per i 
valori profondi della triplicità di Ecate mi limito a rinviare all'analisi di zOGRAFOU (1999), per cui il trimorfismo della dea sarebbe il risultato di una sorta di 'moltiplicazione' della sorveglianza, di 'intensificazione' della sua tutela tramite la ripetizione della sua immagine. Cfr. ora anche ZOGRAFOU (2010), p. 227-248.

24. Su tale opera, ved. FULLERTON (1986); WERTH (2006), p. 35-60; ZOGRAFOU (2010), p. 245-8; assieme a SERAFINI (2012b), p. 181.

25. Cariclide, fr. 1 K.-A.; Eust., ad Il., p. 1197, 29-31 (IV, p. 373, 3-5 V. D. VALK). Il trimorfismo ritorna, con grande insistenza, anche nei papiri magici: ved. ad. es. PGM IV, 2119-25, 2524-30, 2820-26; XXXVI, 189-99; LXX, 16-7.

26. Ved. la bibliografia segnalata da ZOGRAFOU (2010), p. 228.

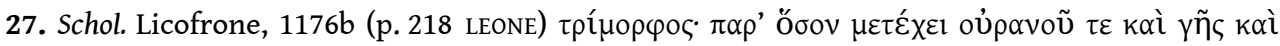
Aǐdov. Cfr. Esiodo, Teogonia, 413-414, 427. Esiodo, infatti, aveva dedicato un'ampia sezione della Teogonia (v. 411-452) alla dea Ecate e ai suoi poteri: su questo passo, cfr. ora SERAFINI (2012), p. 225, n. 3 e 228-229 (con bibliografia), mentre sulla questione del genere letterario, ved. SERAFINI (2011), dove ho proposto la qualifica di 'Lode a Ecate' in luogo dell'abituale (ma impropria) definizione di 'Inno a Ecate' da sempre utilizzata.

28. Sulla fortuna di Ecate in età moderna, ved. SERAFINI (2015): sul trimorfismo, in particolare, cfr. p. 168 e 179-181. Sulla Ecate neo-pagana, invece, ved. SERAFINI (2014b): sull'iconografia contemporanea ved. p. 18-19. Si noti, del resto, che fra le divinità paleovenete preromane è attestata a Làgole (Calalzo) la dea trimorfa Ikate, molto importante nel pantheon paleoveneto, come dimostrano le numerose epigrafi - pubblicate da PELLEGRINI (1950) - e che con ogni probabilità corrisponde a una antica forma di Ecate: si tratta di una divinità 'sanatrice' (sahnate $i$. al dat. = lat. *sanatis /*sanas-itis, «che dà salute», nelle iscrizioni numero 3, 9, 10, 11 di PELLEGRINI), alla quale erano dedicati numerosi ex-voto raffiguranti parti del corpo, e chiamata trumus $\left(\mathrm{nn}^{\mathrm{i}} 2\right.$,

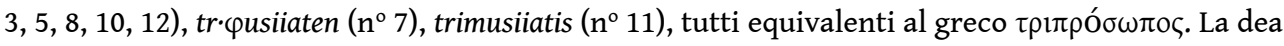
Ikate, inoltre, era anche rappresentata con tre corpi, come dimostra un rilievo trovato in loco: secondo FERRI (1950), che pubblica il manufatto, sarebbero stati gli appellativi riferiti al trimorfismo della dea a crearne l'iconografia. In altre parole, lo studioso esclude che il tipo iconografico sia stato trasmesso direttamente dal mondo greco: si tratterebbe, piuttosto, della resa grafica degli appellativi relativi al trimorfismo della dea. In ogni caso, la presenza di Ecate era già testimoniata con certezza nella situla di Calalzo (PID, no 163), mentre un'iscrizione proveniente dalla Dacia (Apulum) è dedicata alla triformis Libera (CIL III, 1035), ma è assai probabile che qui Libera sia associata a Ecate. Sulla dea Ikate / Ecate, cfr. PELLEGRINI (1951), p. 9194.

29. Esistono due eroi con questo nome: il primo è uno dei figli di Creteo e di Tiro, fondatore ed eponimo della città tessala di Fere (Odissea XI, 259; Apollodoro, I, 9, 11. Cfr. Pindaro, Pitiche IV, 125-126), padre di Admeto, al cui posto com'è noto si rifiutò di morire (Euripide, Alcesti, 12-18. Cfr. Eschilo, Eumenidi, 723-724). Un altro eroe con lo stesso nome è invece un figlio di Medea e Giasone, ucciso dalla madre assieme al fratello Mermero (Apollod., I, 9, 28; Pausania, II, 3, 6; Igino,

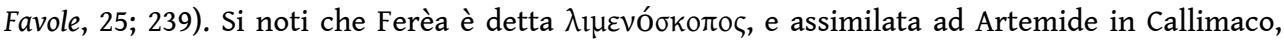
Artemide, 259 e che in PGM IV, 2563 è invece Ecate a essere chiamata $\lambda_{\imath} \mu \varepsilon v \tilde{i} \tau 1 \zeta$ : anche i porti, infatti, sono punti liminali assai significativi. Su Artemide guardiana dei porti, cfr. SERAFINI (2013), p. 150 (con bibliografia).

30. Sull'Inno a Demetra di Callimaco, ved. ora SERAFINI (2015b), mentre su di Erisittone al trivio e sulla possibilità che egli attenda le offerte di cibo destinate alla dea Ecate, cfr. SERAFINI, Povertà.

31. Affrontando il tema della povertà nella sfera religiosa greca, per una discussione approfondita dei fenomeni di furto di cibo sacro, con particolare riguardo anche ai "pasti di Ecate', nonché per un commento dettagliato dei testi qui menzionati, ved. SERAFINI, Povertà.

32. JOHNSTON (1991), p. 219. 
33. Cfr. Schol. Rec. Ar., Pl., 596c, p. 165 Chantry. Lo storico Teopompo testimonia che nella notte di novilunio le statue di Ermes ed Ecate erano incoronate e adornate, in osservanza dei culti tramandati dagli antenati (Teopompo, FGrHist. $115 \mathrm{~F}$ 344). Anche questa informazione è utile per ripetere, seppure indirettamente, la particolarità della notte di novilunio nella dimensione rituale connessa alla dea.

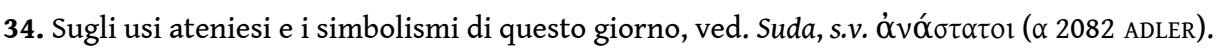

35. Cfr. JOHNSTON (1991), p. 220, n. 14.

36. Sulle offerte a Ecate e la connessione della dea alla dimensione domestica, ved. PARKER (2005), p. 18-20 e 124-126, assieme a FARAONE (2008), p. 210-211 e 222-223.

37. Cfr. ZOGRAFOU (2003), p. 230: «Les triodoi étaient conçus comme des non-lieux pouvant absorber toute sorte d'impuretés». Per quanto riguarda l'eliminazione rituale di rifiuti in Grecia, più in generale, si veda zografou (2005): la studiosa vi esamina i casi in cui l'eliminare qualcosa (che siano resti di purificazioni, oggetti contaminati, o rifiuti 'sacri') può assumere talvolta il valore di una vera e propria offerta.

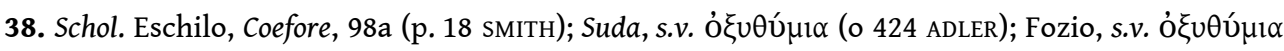
(II, p. 21 NABER); Etym. Magn. 626. 44-46 (s.v. ỏ $\xi v \theta u ́ \mu l \alpha)$. Cfr. Polluce, II, 231 (I, p. 153 BETHE); V, 163 (I, p. 304 BETHE).

39. Già ZOGRAFOU (2003), p. 230 accennava, ma senza sottolinearlo con la dovuta decisione, come tale convergenza non si verifichi nelle fonti più antiche (cfr. anche zOGRAFOU [2005], p. 197, $203 \mathrm{e}$ ZOGRAFOU [2010], p.119-120). Il dato è decisivo e andrebbe evidenziato con più enfasi, poiché permette di seguire lo sviluppo della dea.

40. KASSEL, AUSTIN (1984), p. 128: «albus catulus in sacrificio Triviae, cui nigrum immolatum iri expectares, interrogantis mirationem movet». Sulla preferenza di vittime nere offerte alla dea Ecate, ved. ora zoGRAFou, o.c. (n. 1), p. 267. Si noti anche che in Luciano (Amante delle menzogne, 24), l'epifania della dea Ecate è accompagnata da una muta di cani neri. Uno scoliasta all'Iliade (XXIII, 30) dice che non si sacrificavano ai morti vittime bianche: la consuetudine nel mondo antico era infatti di offrire vittime nere alle divinità infere come anche ai morti o agli eroi (cfr. Odissea XI, 32-33; Virgilio, Georgiche IV, 546; Eneide V, 97). Secondo Arnobio (Adversus Nationes VII, 226), ciò era dovuto alla somiglianza fra la vittima stessa e la divinità cui era destinata, mentre Plinio ( Storia Naturale XXX, 21) testimonia infine che cani neri erano sacrificati dai maghi per purificare le case e avevano valore profilattico contro le maledizioni. Cfr. infine anche G. D'ANNUNZIO, Fedra, Atto I, scena 1, v. 1146-1148: «SCHIAVA TEBANA: Son bianca. / Nera vittima chiedono / gli Inferi. FEDRA: Ecàte è pallida». Si noti che l'ultimo verso ricalca il Hecate pallenti di Lucano (Farsalia VI, 737).

41. Sul rituale descritto in questo passo delle Argonautiche Orfiche, ved. OGDEN (2001), p. 172.

42. Sui significati e i rituali che prevedono lo scavo di un bothros, ved. soprattutto EКROTH (2002), p. 60-74.

43. Plutarco, Quest. Rom., 280c: «Tutti i Greci utilizzavano, e alcuni ne utilizzano ancora oggi, il cane come vittima per le purificazioni. Assieme a tutte le altre offerte purificatorie, essi portano a Ecate dei cani, e per mezzo di questi purificano chi ha bisogno di essere decontaminato: essi chiamano questo genere di purificazione 'immolazione del cagnolino'».

44. Si noti che il cane era prescritto in certe diete terapeutiche ippocratiche: Hipp., Vict., 79, 82, infatti, raccomanda cane arrosto per alcune condizioni mediche. Tuttavia, occorre ricordare che Porfirio, Astinenza I, 14, 3 afferma che i Greci evitavano carne di cane, cavallo e asino perché questi erano ritenuti animali domestici. Cfr. anche Plinio, XXX, 42-43 e 64, per cui i cuccioli di cane sono considerati adatti ai rituali di purificazione poiché possono facilmente assorbire i mali e le malattie ai quali sono esposti.

45. Poco dopo, lo stesso Plutarco (Quest. Rom., 290d) conferma tale associazione, attestando l'esistenza di un rito di purificazione in Beozia che consisteva nel passare fra le due metà di un 
cane squarciato: su questo rito, ved. MAINOLDI (1981), p. 33, mentre sul culto di Ecate in Beozia, ved. ora SERAFINI (2013c). Inoltre, anche la lustrazione primaverile dell'esercito macedone avveniva passando fra le due metà di un cane sacrificato e poi smembrato, le cui due parti erano poste ai lati di una strada attraverso la quale doveva sfilare, secondo un rigido ordine gerarchico,

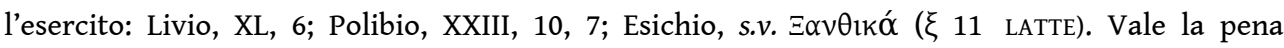
ricordare anche quanto descritto da Erodoto (VII, 39), il quale racconta che l'esercito persiano fu costretto a passare fra le due parti del figlio di Pizio tagliato a metà: il tutto è presentato come una punizione di Serse, ma non è da escludere che dietro a questa azione possa essere presente un qualche costume rituale vero e proprio.

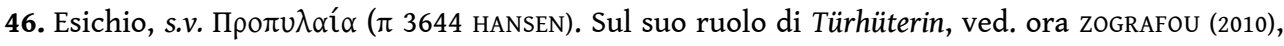
p. 93-109.

47. Non è questa la sede per una discussione topografica dettagliata sull'argomento. Mi limito a ricordare che a Selinunte era presente un recinto dedicato a Ecate accanto al propylon del santuario della Malophoros (ved. SERAFINI, Selinunte); a Eleusi, invece, di fronte ai Grandi Propilei, si trovava il naos di Artemide Propylaia (Paus., I, 38, 6), identificabile con Ecate. Sia l'epiclesi di Propylaia, sia il ruolo giocato da Ecate nell'Inno Omerico a Demetra hanno infatti condotto giustamente svariati studiosi a identificare l'Artemide di Eleusi con Ecate: cfr. SERAFINI (2012), p. 230 e SERAFINI (2013b), p. 12, assieme al già menzionato SERAFINI, Selinunte (con bibliografia).

48. Cfr. KRAUS (1960), p. 86: «Eine feierliche Anrufung, deren ganze Macht auch noch aus dieser einzigen Zeile fühlbar wird».

49. Come aggettivo è attestato ad esempio in Bacchilide (Epinici VI, 14), in cui si parla di

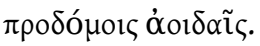

50. Schol. Ar., Vespe, 804 b (p. 129 KOSTER). Cfr. anche schol. Ar., Rane, 366b (p. 72 CHANTRY).

51. Ved. HENDERSON (1987), p. 74-75, ma soprattutto la dettagliata analisi di DORATI (2002), p. 85-

87.

52. Schol. Vet. Ar., Rane, 366b e 366c (p. 61-62 CHANTRY); Schol. Rec. Ar., Rane, 366a (p. 72 CHANTRY).

53. Cfr. Ar., Rane, 153; Ar., Donne all'assemblea, 330; e Lisia, fr. 73 THALHEIM.

54. Cfr. a tal proposito l'espressiva testimonianza di Olymp. Phil. Plat. Phedon. C III $\lambda \alpha^{\prime}$ (p. 192

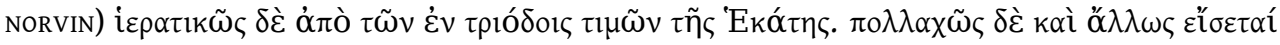
$\tau \imath \zeta$.

55. Diversa l'ipotesi di PARKER (2005), p. 414-415 il quale pone piuttosto l'accento sul pericolo costituito dalla dea stessa: «Hecate is honoured at the door of the house to discourage her from stepping inside and that form of intimacy which is cultivated with her is a way of making the threat she poses psychologically manageable».

\section{RIASSUNTI}

La déesse Hécate est une déesse intimement liée aux lieux de passage. Son caractère liminal, qui se traduit par un lien étroit avec les carrefours et les portes, se manifeste en une grande variété de formes cultuelles. Le présent article analyse cette relation à la lumière des témoignages littéraires qui attestent une série de rituels accomplis en son honneur aux carrefours: de l'offrande de nourriture durant la nuit de la nouvelle lune (les "repas d'Hécate ») jusqu'aux sacrifices de chiens à la déesse, en passant par le dépôt des débris provenant de rites de purification. En outre, Hécate se situe souvent aux entrées : déesse apotropaïque, elle protège la 
maison de dangereuses influences provenant de l'extérieur. En résumé, cette contribution vise à offrir un parcours diachronique et exhaustif des sources littéraires attestant le lien de la déesse avec les lieux de passages.

Hecate is a goddess closely connected to the passageways. Its liminality results in a strong connection both with crossroads and gates: this relationship shows itself in a large variety of ritual manifestations. This paper analyzes that connection by checking the literary evidence, which documents plenty of rituals performed in his honor at the crossroads: firstly, the offering of food during the night of new moon (the so-called "meals of Hecate"), secondly, the sacrifice of dogs to the goddess, and finally the deposition in the triodoi of the rejects that remained after the household rites of purification. This goddess is also placed often by the entrance of the houses: she is an apotropaic deity, which protects the inside from the dangerous influences that come from outside. Thus, the paper attempts to offer a complete, and organically arranged overview of all the literary sources on this subject.

\section{AUTORE}

NICOLA SERAFINI

Università di Urbino

DISCUM

Via Sant'Andrea 34

I-61029 Urbino

nicola.serafini@me.com 